Corresponding Author:

Lee Khai Loon

leekhailoon@ump.edu.my

Received: 5 August 2019

Accepted: 14 August 2019

Published: 18 August 2019

Publishing services provided by

Knowledge E

(c) Lee Khai Loon and Lim Cean Peing. This article is distributed under the terms of the

Commons Attribution License,

which permits unrestricted use

and redistribution provided that

the original author and source

are credited.

Selection and Peer-review under the responsibility of the FGIC2019 Conference Committee.

\section{Big Data and Predictive Analytics Capabilities: A Review of Literature on Its Impact on Firm's Financial Performance}

\section{Lee Khai Loon and Lim Cean Peing}

Faculty of Industrial Management, Universiti Malaysia Pahang, Lebuhraya Tun Razak, 26300 Gambang, Pahang, Malaysia

\section{Abstract}

In the era of the fourth industrial revolution, big data and predictive analytics (BDPA) capabilities considered as one of the significant resources that enable a firm to gain competitiveness. Nowadays, the advancement of technology information increases the difficulty of the firm to manage vast amounts of structured and unstructured data. The excessive growth between data captured and the firm's capabilities to manage, process, analyze, and transfer the big data to actionable knowledge and value still challenges many firms in the competitive market. Besides, the lack of tangible resources, technical skills, management skills, organizational learning, and data-driven culture are some of the challenges for firms to apply analytics approach to support the data processing process. These situations led to poor decision making by the firm and result in high operation cost and lower profitability. However, the situation will be different if the firm able to manage BDPA capabilities in the right way. Based on the identified problems, this study aims to review the impact of BDPA capabilities on a firm's financial performance. This study is expected to enhance the body of knowledge on BDPA capabilities and the firm's financial performance. This study also provides information regarding the importance of BDPA capabilities on the firm's financial performance to industrial practitioners. An empirical study on this subject matter is suggested for future researchers, especially in Malaysia manufacturing industry.

Keywords: big data analytics, predictive analytics, big data and predictive analytics capabilities, firm's financial performance.

\section{Introduction}

Big data refers to the techniques, practices, methodologies, and applications related to the acquisition, analysis, storage, and integration of massive amount of data to support business decision making (Mikalef, Pappas, Krogstie, \& Giannakos, 2017). According to Jeble et al. (2018), big data and predictive analytics (BDPA) is an emerging field where it is adopted with various computer algorithms and statistical techniques to obtain insight and patterns from big data sets. Recently, many firms recognized BDPA as one of the 
significant resources to gain competitiveness. According to Jeble et al. (2018), the raising of digitalization in every aspect of the business has made BDPA ranked as an essential item for the senior executives in the organizations, no matter for the improvement of firm's financial performance or firm's competitiveness. The advancement in new technology information such as the emerged of smartphones, cloud computing, digital devices, scanning devices, and the Internet of Things (IOT) enable the firms to discover new information and identify a new pattern that enables the firms to make improvements in their processes and lead to an improvement in productivity. The emerging of BDPA transformed the way the firms run their business (Akter, Wamba, Gunasekaran, Dubey, \& Childe, 2016). Other than that, the new technology information enables the firms to generate variety and a massive amount of big data sets and also enhances the process of the firms in creating data analytics capabilities.

Within highly competitive business environments and rapidly changing in customer's demand, many firms are obliged to be more quickly and boldly to experiment to survive and to maintain competitiveness in the market. Popovič, Hackney, Tassabehji, and Castelli (2018) highlighted that organizations should focus on a keep changing or uncertainty business environment at the right time and use the appropriate method in doing business. To achieve that, firms should continuously identify and develop new advantages to ensure the ability to create new opportunities effectively and to exploit new opportunities efficiently as well as to improve the firm's financial performance. Wamba, Akter, Edwards, Chopin, and Gnanzou (2015) stated that "collecting, storing, and big mining data for insights can provide significant value for the companies to enhancing productivity and competitiveness." Besides, Wamba et al. (2017) further stated that BDPA is the application of big data, analytical tools, and techniques to provide a solution to the firms in obtaining insights. Also, Jeble et al. (2018) indicated predictive analytics is a useful method used to identify and collect meaningful data which can be used to determine data pattern by applying the application of various data analysis techniques which including data pattern recognition, data mining, statistics, and artificial intelligence.

Profits maximization, increases market share, and fulfill their customers' requirements considered as the primary goal of every firm. Globalization, the arrival of social media, and technology advancement lead to the competition of firms to become more critical. Thus, to achieve those goals, all firms are essential to have BDPA capabilities. Akter et al. (2016) stated: "if a particular company that does not adopt a big data and predictive analytics (BDPA) strategy, then they are facing risk to lose their market share and momentum in competing with other companies in the market." Therefore, the 
investment in BDPA is one of the methods in ensuring the firms' sustained competitive advantage. Jeble et al. (2018) noted that firm integration and deployment of BDPA strategic resources able to gain competitive advantages from competitors. Jeble et al. (2018) further revealed that firms should focus on tangible resources, technical skills, management skills, organizational learning, and data-driven culture in studying BDPA (Jeble et al., 2018).

Recently, some academics have stressed the low financial performance of some particular firms is related to poor decision making. In the manufacturing industry, poor decision making will lead to high operational cost and lower profitability (Raguseo \& Vitari, 2018). While Lee, Kweon, Kim, and Chai (2017) mentioned, the manufacturer could not enhance their financial performance, if the firms do not have better managerial decision-making. Besides, Akter et al. (2016) indicated that the firms do not adopt a BDPA strategy will losing market share and momentum due to the lack of advantages in knowing customers demand. Many manufacturing firms all over the world, including Malaysian manufacturing firms, are facing the same problems, which are weak firms' performance, especially in their financial performance. According to Lee et al. (2017), the reasons that many firms are facing these kinds of problem is because they are unable to manage vast amounts of unstructured data. For instance, the existing data analytics tools unable to effectively extract valuable and meaningful data from those unstructured data, lack of expertise in the big data management field, and lack of support of top management in BDPA project investment.

Furthermore, the excessive growth between data captured and firm's capabilities to process, manage, analyze, and transfer big data flow to actionable knowledge and value still challenges the firms to be competitive (Garmaki, Boughzala, \& Wamba, 2016). Also, the lack of knowledge, supportive management, human resources skills, organizational culture to share data, and insufficient technology are some of the challenges for firms to apply analytics approach to support the data processing process (Garmaki et al., 2016). Considering the development in BDPA in several fields, there has been a trend of research in the relationship of big data and firm's financial performance in the manufacturing industry ( Jeble et al., 2018). However, current literature focused on BDPA capabilities on firms' social, environmental, and economic performance. Therefore, Côrte-Real, Oliveira, and Ruivo (2017) argued that there is still a lack of finding on the relationship between BDPA capabilities on firms' financial performance. Furthermore, there is still insufficient evidence showing that the actual impact of BDPA capabilities on firm financial performance(Lee et al., 2017; Mikalef et al., 2017), Besides, the assessment of the real value and effect of big data on firms' financial performance remains 
vague (Garmaki et al., 2016). Hence, this research aims to review the impact of BDPA capabilities on the firm's financial performance.

\section{Literature Review}

\subsection{An overview of big data}

The concept of big data generating great attention worldwide and emerged a new filed of analytic. Akter et al. (2016) defined big data as a massive amount of diverse observational data, while Jeble et al. (2018) defined big data as "any collection of large and complex data sets that are difficult to store, process and analyses with earlier methods." It is focused on the uses of computer science, advanced data storage, and management techniques and statistics concepts to collect and extract the meaningful value from the big data. Akter et al. (2016) further indicated that there are five "Vs" that can represent the big data which are volume, velocity, variety, veracity, and value. In addition, Opresnik and Taisch (2015) provided a simple definition for the five big data characteristics: 'volume' refers to the data generated from the machine; 'velocity' refers to the reflect to the speed and frequency of the transactions; 'variety' refers to the increase of structured or unstructured data created by different sources; 'value' is concerning about the value extracted from the existing data. According to Lee et al. (2017), big data is a high volume, velocity, and variety of information assets. It enables the firms to enhances insight and better decision making by innovative forms of information processing in a cost-effective way.

\subsubsection{Big data analytics}

Nowadays, large-scale data are created by many online applications, such as online banking, e-commerce, and e-transactions. The emerged of big data, and the advancement in analytic techniques created opportunities to the firms, these opportunities enabled the firms to increases their competitiveness level ( Jeble et al., 2018). According to Lee et al. (2017), big data analytics (BDA) known as one of the emerging information system, where the implementing of BDA will help firms to achieve their excellent performance. Ghasemaghaei, Hassanein, and Turel (2015) defined BDA as the applications of tools or processes to extract large-scale and disperse data sets for obtaining meaningful insights that can help to improve organizational performance. While, Lamba and Dubey (2015) defined BDA as the use of various analytic methods to gain useful information 
from large data sets such as provide the firms actionable descriptive, predictive, and prescriptive results. The study of Wamba et al. (2017) stated that BDA forms a clear barrier between low and high performing organizations, as it allows firms to look forward, enhances revenue, reduces cost, and increases firms competitiveness. In the era of technological advancement, many firms now are not concerned with descriptive analytic such as what happened or why it happened, but they are focusing on predictive analytics such as in present what is happening or in the future what is likely to happen (Jeble et al., 2018).

\subsubsection{Predictive analytics}

To know what would happen in the future, firms are necessary to have predictive analytics. This is because predictive analytics can get insights from the existing big data. According to Jeble et al. (2018), to predict some things that will happen in the future, predictive analytics is a useful technique that capable of answering this question. Also, Abbott (2014) defined predictive as a process that extracting meaningful data patterns through pattern recognition techniques, artificial intelligence, machine learning, statistics, and data mining. Also, Jeble, Kumari, and Patil (2016) indicated that predictive analytics also referred to the advanced analytic technique, where it is an application of analytic data techniques to help the organizations to answer certain questions or solve some of the problems. Thus, predictive analytics enable organizations to do better decision making and enhance firms performance (Jeble et al., 2016).

\subsection{Big data and predictive analytics capabilities}

Big data and predictive analytics (BDPA) capabilities are defined as a higher-order of organizational capabilities where it depends on the bundling of strategic organizational resources (Wamba et al., 2017). Akter et al. (2016) stated that BDPA capabilities affect organizational performance. Moreover, Dubey et al. (2017) indicated that the BDPA could be conceptualized as capabilities where these capabilities are essential to a firm. This is because, without BDPA capabilities, the firms are unable to gains useful information from the big data sets.

Besides, effectively development on organizational BDPA capabilities may lead to achievement in competitive advantage and the improvement of firms' performance (Dubey et al., 2017). According to Jeble et al. (2018), when firms able to assemble, integrate, and allocate their big data resources, then the firms will develop their BDPA 
capabilities which opposite from their opponents. Additionally, Jeble et al. (2018) called this ability as the BDPA capabilities where these capabilities enable the firms to perform better in the process of collecting, analyzing, and processing big data.

Dubey et al. (2017) highlighted that the BDPA capabilities are referred to the combination of the firms' resources such as human resources, technical skills, and managerial skills to create an ability to increase competitiveness. However, Jeble et al. (2018) have a different view in which resources can be categories as tangible and intangible resources. Therefore, in this study, the resources that needed to developing BDPA capabilities are tangible, technical skills, management skills, and intangible resources (i.e., organizational learning and data-driven culture).

\subsubsection{Tangible resources}

According to Jeble et al. (2018), tangible resources referred to an organizations' building, information technology infrastructure, capital, networks, connectivity, and data sources. Besides, Dubey et al. (2018) reported that tangible resources in BDPA capabilities as those things that can be sold or bought from markets such as capital, building, and machinery. It is necessary to have these resources to enable the firms to develop analytic solutions. However, these resources are not capable of generating a competitive advantage to the firms if the firms are not acquired with the foundation required to build capabilities (Jeble et al., 2018).

Investment in big data and predictive analytics (BDPA) project are significant to the firms. Investment in BDPA project is substantial because it allows the firms to extract useful information effectively. Besides that, Dubey et al. (2017) stated that BDPA project is considered as a long-term investment where it requires devote some time to get back the return of investment. However, this investment will assure that the firms can have a significant improvement in their firms' performance (Dubey et al., 2017).

\subsubsection{Technical skills}

According to Dubey et al. (2017), technical skills can be defined as the knowledge that requires the application of new technology or algorithms to extract significant value from big data. Jeble et al. (2018) stated that BDPA capabilities normally required big data analyst to have specific skills and knowledge such as statistical analysis, machine learning, business acumen to provide solutions of solving business problems, problemsolving skills, and strong communication and soft skills. Jeble et al. (2018) further noted 
that technical skills could develop by several methods, such as hiring a new analyst or by providing employees training.

Besides, technical skills also can be referred to the competencies in extracting data, data cleaning, and understanding programming tools (Gupta \& George, 2016). On the other hand, Akter et al. (2016) mentioned that technical skills refer to knowledge related to technical elements which including operational systems, the language of programming, statistic, and database operating systems. Without doubt, technical skills in big data technology are critical to the firms. Gupta and George (2016) noted that firms with big data and predictive analytics skilled employees are likely to have a more competitive advantage as compared to the firms that do not acquire big data and predictive analytics skilled employees. The above discussion has been highlighted in the recent study by Anwar, Khan, and Shah (2018): "when the firms acquired with the knowledge related to the big data, they will have the ability to extract information related to markets, customers, and consumers from big data, and where this information will enable the firms to gauge external trends and changes".

\subsubsection{Management skills}

Management skills considered as crucial elements for BDPA capabilities, where managers are playing a significant role in leading, managing, and controlling all resources included in big data and predictive analytics capabilities such as manage human resources, financial capital as well as create a data-driven culture in the organization. This is because management skills are rare, unique, and differentiated (Jeble et al., 2018).

According to Dubey et al. (2017), the success of BDPA projects is relying on managers' decision making. For example, how well a manager can create or combine his or her team members from the different functional unit with the right skills and lead his or her team members to achieve the organization's goals. On the other hands, it is considered that the intelligence gathered are pointless if managers fail to understand the significant value from the extracted insight (Gupta \& George, 2016). Therefore, the managers should have the abilities to understand and able to make the right decision at the right time through the information extracted.

Indispensably, good communication skills and the ability to create relationship are crucial to managers to deal with internal and external stakeholders for analytic projects. A good relationship between big data managers with other functional managers in 
working will lead to better development in human big data skills, and this also leads to the improvement in firms' performance.

\subsubsection{Intangible Resources}

Generally, intangible resources are known as the center of a firm's performance, especially in dynamic market situations (Dubey et al., 2017). Intangible resources are dissimilar to the tangible resources where intangible resources have not clear or visible boundaries, and its value is highly depended on context (Gupta \& George, 2016). According to Dubey et al. (2018), intangible resources cannot be easily sold or bought in the market like most tangible resources. This is because intangible resources are untraceable unique, rare, valuable, and irreplaceable (Gupta \& George, 2016). Hence, this study follows Jeble et al. (2018) study in which the intangible resources are categorized into two aspects, which are organizational learning and data-driven culture.

\subsubsection{Organizational learning}

Nowadays, organizational learning is an important aspect, especially to react to the uncertainty market environment (Dubey et al., 2018). The uncertainty environment that firms have to face are such as the economic, social, environmental, technological, political, or legal environment changes. According to Dubey et al. (2017), due to the emergence of new technologies, the employees' knowledge may become outdated if they do not follow the speed of technology advancement. Jeble et al. (2018) stated: "it is crucial for a firm to ensure their employees are updated with the latest knowledge related to their field." This statement supported by Dubey et al. (2018): "continuously upgrade the knowledge of employees will increase the ability of firms compete in the dynamic market place." Gupta and George (2016) mentioned, the intensity of a firm to learn, firms are likely to get more advantages than its rivals when competing in the same market. This is because a firm can combine its employees' knowledge and the insights extracted from big data to make better performance. Moreover, Jeble et al. (2018) also stated there are two methods to improve the firm's performance which are learned faster than their competitors and ensured the firm keep pace in the changes of the dynamic situations. Thus, it is clear that organizational learning is one of the important resources needed to create BDPA capabilities to improve the firm's performance. 


\subsubsection{Data-driven culture}

Commonly known that the difficulty to change organizational culture is facing by most of the organization. According to Gupta and George (2016), it is difficult to understand and describe the concept of a data-driven culture. Over the past few years, several works of literature have shown inconsistent meaning across data-driven culture or organizational culture. According to Arunachalam, Kumar, and Kawalek (2018), datadriven culture is an intangible thing where it is related to the organization employees' belief, attitudes, and opinion towards data-driven decision making. Dubey et al. (2018) argued that data-driven culture might be an essential key for a firm to gain improvement in their firm's performance. Some scholars have argued that organization culture or datadriven culture can enhance or restrain organization's ability to gain benefit from the big data (Arunachalam et al., 2018; Dubey et al., 2018; Gupta \& George, 2016). Also, Gupta and George (2016) reported that data-driven culture is a crucial element that allows firms to enhance success in BDPA initiatives. Firms' BDPA projects often ineffective are highly related to an organizational culture that refused to change (Gupta \& George, 2016). Thus, it is important that all top management and employees at all levels and the different functional department understand and believe in the potential of BDPA capabilities. Otherwise, the effort taken by the firm to extract valuables information from big data will be going meaningless.

\subsection{Firm's financial performance}

Firm performance can refer to a firm's ability to gain new customers and retain existing customers, increase sales, enhance profitability, and improve return on investment (Wamba et al., 2017). With technology advancement, BDPA is one of the emerging information systems where it enables the firms to enhance their performance efficiently and effectively. According to Lazăr (2016), firm resources such as firm's assets, capabilities, information, knowledge, and organizational processes are the important resources where those resources allow the firm to execute and implement organizational strategies that help the firm to enhance efficiency and effectiveness. In enhancing efficiency, Hassna and Lowry (2016) noted that a firm should reduce the cost when adaptation into a new market such as reducing the costs of producing a product or service. In the other hand, the firm can enhance effectiveness through enabling a firm to snatch new opportunity, like improving a product or service (Raguseo \& Vitari, 2018). 
Generally, financial performance is referred to as the improvement in economic goals. Financial performance measures by profitability, customer retention, overall financial performance (Raguseo \& Vitari, 2018; Wamba et al., 2017), sales growth, and return-oninvestment (Yu, Chavez, Jacobs, \& Feng, 2018). With the growth of big data technologies, firms are expected to enhance competitive advantages over their rivals from big data analytic solutions (Raguseo \& Vitari, 2018). Therefore, firms must have BDPA capabilities, especially in the dynamics markets. It is because the managers have to deal with many factors and issues increasing in the markets. Jeble et al. (2018) stated that the firm's manager spends much time in solving issues such as to get advantage from cost and quality. Cost and quality are one of the critical aspects to measure financial performance. Therefore, firms are sourcing their materials globally. One of the methods to get cost and quality advantage is firmed choose to import large quantities of raw materials to get cost-saving. However, there are some uncertainty conditions related to firm's operational which firm's managers have to deal with such as the number of suppliers, delivery lead time, and different geographic locations (Jeble et al., 2016).

Hence, to deals with such uncertainty conditions, firms are requiring to have BDPA capabilities. BDPA capabilities will enable the firms to enhanced insight, decision making (Raguseo \& Vitari, 2018), delivery status, and real-time demand (Jeble et al., 2018). Thus, with BDPA capabilities, it is possible that firms can achieve their economic goals where they have a clear path on how to increase their profitability, sales growth, return on investment, and customer retention.

\subsection{The impact of BDPA capabilities on financial performance}

Raguso and Vitari (2018) defined financial performance as the capabilities of the firm to use its resources to enhance and achieve its goals which are to improve a firm's revenue, profitability, sales, return-on-investment, and operational cost. BDPA capabilities are known as a base for a firm to gain a competitive advantage. However, it depends on how the firms extract meaningful and valuable information at the right moment (Raguseo \& Vitari, 2018). According to Anwar et al. (2018), BDPA capabilities are an important key that will lead to firm efficiency and better decision making, where it processes valuable information to a firm. According to Mikalef et al. (2017), with valuable information, a firm's managers can manage their operational process efficiently. The managers can use the valuable information provided by BDPA to make better decisions such as how many quantities of products should be produced when to deliver the products, which supplier has a better price of materials, and in future what would happen in customers' demand. 
Furthermore, Yu et al. (2018) reported that BDPA capabilities enable firms to achieve significant improvements in their firm's financial performance such as specific product development based on customer data, forecasting demand, and lean operations and production. Without a doubt, all of these are highly related to decision making on how to reduce cost and increase in profit. Therefore, as stated by Selvam, Gayathri, Vasanth, Lingaraja, and Marxiaoli (2016), the right decision made by the firms will lead to the improvement in their performance especially the aspects related to the cost. A review of the literature showed that BPDA capabilities are closely related to firm's financial performance (Anwar et al., 2018; Mikalef et al., 2017; Raguseo \& Vitari, 2018; Yu et al., 2018).

According to Akter et al. (2016), the firm's BDPA capabilities are believed to affect firm financial performance. BDPA capabilities would also lead to higher financial performance. To achieve higher financial performance, BDPA capabilities of the firms are playing an important role where it allows the firm's manager to have better decisionmaking. For instance, BDPA capabilities allow the firm's managers to make the better decision on price optimization and profit maximization (Akter et al., 2016), increase sale, getting more new customers, improve return on investment, and enhance profitability (Raguseo \& Vitari, 2018; Wamba et al., 2017). Moreover, Srinivasan and Arunasalam (2013) showed that a firm could enjoy benefits by cost reduction through BDPA capabilities such as reduces the amount of waste and re-work and improved in product quality. On the other hand, lack of knowledge and skills in BDPA could result in making an incorrect analysis or decision (Ferraris, Mazzoleni, Devalle, \& Couturier, 2018). Incorrect analysis of big data will provide the wrong information to the firm, and it also will cause an increase in operational cost (Anwar et al., 2018; Mikalef et al., 2017). Hence, it is important to have BDPA capabilities to enable the firm's managers to make a better decision on how to reduce their operational cost and increase the level of financial performance.

Tangible resources can be utilized effectively and efficiently to develop different capabilities in BDPA (Dubey et al., 2018). In this study, tangible resources are those that visible such as physical and financial assets of the organization. Tangible resources can be represented by basic resources, data, and technologies. According to Gupta and George (2016), basic resources such as capital and time of the firm is an important element to achieve the best financial performance. This is because when the firms are investing enough time and money into their BDPA capabilities, they can grasp the high volume and variety of data that are rapidly increased due to the technology 
advancements. By control over the high volume and variety of data, the firms make important decisions on how to improve their financial performance.

On the other hand, tangible resources are necessary for a firm's analyst to develop and find analytics solutions (Jeble et al., 2018). The investment into BDPA capabilities and related technologies not only provide a competitive advantage to the firm, but it also enables the firm to develop strong IT infrastructure to gather, analyze and use the big data sets for making operational decisions and predictions for the plan. These help the firm to improve its profitability because of less error and mistake in the firm's operations.

Besides, technical skills are one of the dimensions included in the BDPA capabilities. According to Jeble et al. (2018), to ensure the efficiency and effectiveness of the firm BDPA projects, it is crucial to have technical skills because BDPA technologies normally required an employee to acquire with specifics knowledge and skills related to BPDA. Without the specific knowledge and skills such as statistical analysis, machine learning, and business acumen, the employees are unable to run the BDPA projects effectively. Ferraris et al. (2018) supported the statement given by Jeble et al. (2018) and further highlighted that effective BDPA projects would supply the top management correct and accurate information. This information will help top management to make a better decision on what to do in the next step. For instance, how many products should produce, what kind of product is most preferable by consumers in the market, and what is the trend of consumer pattern. It is clear that top management should have excellent management skills. This is because the success of BDPA projects depends on the managers' managerial skills to develop a team with the right skills and aligns the team members with achieving the goals of the firm. According to Wamba et al. (2017), a good manager with the right skills can make the correct decision to improve the firms' performance through the information extracted by the team members. As stated by Gupta and George (2016), "a firm with skilled employees in the big data field are likely to have an advantage over its competitors. However, this advantage cannot be achieved without the managers who will respond to manage such skilled employees and BDPA project". Therefore, technical skills and management skills positively link with the firm's financial performance.

According to Dubey et al. (2017), there is a positive relationship between intangible resources and the firms' financial performance. To achieve excellent financial performance, the firms must be able to compete for the market. Based on Jeble et al. (2018), they have mentioned: "the only way to retain firm competitiveness is to learn faster than the other competitors." Nowadays, there are nonstop changing in the external 
environment, such as changes in the economic, social, political, and legal environment. According to Gupta and George (2016), the firms can achieve the best financial through their employee's continuous learning process, and this statement is supported by Dubey et al. (2017). They stated: "the firms have the propensity to provide their employees to learn knowledge related to BDPA technologies may remain their competitiveness." Hence, when the firms have competitiveness to compete in the market, it is clear that financial performance can be enhanced. Besides, the organizational learning and datadriven culture is another one important dimension that will help to enhance the firms' financial performance. Based on Jeble et al. (2018), data-driven culture is a source of competitive advantage for a firm. This is because the data-driven culture can enable the firm to extract benefit from big data. According to Childe et al. (2018), when the management and employees of the firm at all functional level believe and able to utilize the information extracted from big data effectively, this will help to improve the firm's financial performance. Therefore, the review of the literature showed that organizational learning and data-driven culture able to improve the firm's financial performance.

\section{Recommendations for Practitioner and Researcher}

Based on the literature reviews to date, many firms are setting their goals to fulfill their customer requirements, increase sale, increase profitability, and gain competitive advantages from their competitors. However, to achieve these goals, many firms facing similar problems which are unable to identify the actual demands or the requirement from the customers. A part of the reason is due to the lack of a database for data analysis.

Undeniably, the investment in BDPA capabilities will bring benefits to all industries, especially for the manufacturing industry. Some recent studies provide suggestion to firms in which the investment on BDPA could provide a clear overview of firm's competitive landscape in the respective industries and further leading the firm to achieve competitive advantage (Akter et al., 2016; Côrte-Real et al., 2017). With good BDPA capabilities, the firm can increase the accuracy of forecasting and minimizing inventory cost as well as lead to expanding the market area or market growth (Lee et al., 2017). Besides, the implementation of BDPA also increases the ability of organizations' talent to capture a vast amount of big data sets in making a robust decision (Garmaki et al., 2016). Good decision making of organizations will help in expanding the market. Hence, future research is suggested to focus on the empirical study of the relationship between 
BPDA capabilities and the firm's financial performance in the Malaysia manufacturing industry.

From a practical perspective, this study aims to increase the knowledge and awareness of the practitioner on the importance of BDPA capabilities to the firm. Given the evidence of the literature review, the investment on BDPA capabilities able to bring a significant impact to the firm's financial performance. Therefore, increase the use of BDPA should start to build the awareness of relevant employee on the importance of BDPA capabilities. Besides, the firm could start to invest in BDPA capabilities, which include tangible resources, provide training to build employees' technical skills, and management skills. Also, intangible resources, organizational learning, and data-driven culture are equally important elements in BDPA capabilities. Some firms are a focus on tangible resources, while the intangible resources are critical elements to run tangible resources such as continuously sending employees for learning to ensure that they are updated with new knowledge and skills. Last but not least, the successful and effective BDPA capabilities investment must obtain the cooperation between the top management, the employee at all levels and the different functional department must work closely with the same beliefs on the potential of BDPA capabilities on firm's financial performance.

\section{Concluding Remarks}

This study was conducted to understand the impact of BDPA capabilities on the firm's financial performance. The findings from the review of the literature revealed that BDPA capabilities which consist of tangible resources, technical skills, management skills, organizational learning, and data-driven culture play an important role in improving firm's financial performance. The power of BDPA capabilities hinges to a large extent on the ability of the industry to operate more efficiently and effectively towards the firm's financial performance and further improve competitive advantage. This study is expected to enhance the body of knowledge on BDPA capabilities and the firm's financial performance. This study also provides information regarding the importance of BDPA capabilities on the firm's financial performance to industrial practitioners. This study has reviewed and discussed the BDPA capabilities in a brief conceptual study. Therefore, further research is advised to target more comprehensively in the empirical study to test and proof the relationship between BDPA capabilities and the firm's financial performance, as shown in the conceptual framework in this study. As 
mentioned earlier, Malaysia manufacturing industry is potential to be investigated on this subject matter.

\section{Acknowledgement}

We would like to thank Faculty of Industrial Management and FIM's Governance and Integrity Centre, Universiti Malaysia Pahang for the financial support by sponsoring this paper to be presented in the FGIC 2nd Conference on Governance and Integrity 2019.

\section{References}

[1] Abbott, D. (2014). Applied predictive analytics: Principles and techniques for the professional data analyst: John Wiley \& Sons.

[2] Akter, S., Wamba, S. F., Gunasekaran, A., Dubey, R., \& Childe, S. J. (2016). How to improve firm performance using big data analytics capability and business strategy alignment? International Journal of Production Economics, 182, 113-131.

[3] Anwar, M., Khan, S. Z., \& Shah, S. Z. A. (2018). Big Data Capabilities and Firm's Performance: A Mediating Role of Competitive Advantage. Journal of Information \& Knowledge Management, 17(04), 1850045.

[4] Arunachalam, D., Kumar, N., \& Kawalek, J. P. (2018). Understanding big data analytics capabilities in supply chain management: Unravelling the issues, challenges and implications for practice. Transportation Research Part E: Logistics and Transportation Review, 114, 416-436.

[5] Childe, S. J., Dubey, R., Gunasekaran, A., Luo, Z., Wamba, S. F., Roubaud, D., \& Foropon, C. (2018). Examining the role of big data and predictive analytics on collaborative performance in context to sustainable consumption and production behaviour. Journal of Cleaner Production, 196, 1508-1521.

[6] Côrte-Real, N., Oliveira, T., \& Ruivo, P. (2017). Assessing business value of Big Data Analytics in European firms. Journal of Business Research, 70, 379-390.

[7] Dubey, R., Gunasekaran, A., Childe, S. J., Papadopoulos, T., Luo, Z., Wamba, S. F., \& Roubaud, D. (2017). Can big data and predictive analytics improve social and environmental sustainability? Technological Forecasting and Social Change.

[8] Dubey, R., Luo, Z., Gunasekaran, A., Akter, S., Hazen, B. T., \& Douglas, M. A. (2018). Big data and predictive analytics in humanitarian supply chains: enabling visibility and coordination in the presence of swift trust. The International Journal of Logistics Management, 29(2), 485-512. 
[9] Ferraris, A., Mazzoleni, A., Devalle, A., \& Couturier, J. (2018). Big data analytics capabilities and knowledge management: impact on firm performance. Management Decision.

[10] Garmaki, M., Boughzala, I., \& Wamba, S. F. (2016). The effect of Big Data Analytics Capability on Firm Performance. Paper presented at the PACIS.

[11] Ghasemaghaei, M., Hassanein, K., \& Turel, O. (2015). Impacts of big data analytics on organizations: a resource fit perspective.

[12] Gupta, M., \& George, J. F. (2016). Toward the development of a big data analytics capability. Information \& Management, 53(8), 1049-1064.

[13] Hassna, G., \& Lowry, P. (2016). Big Data Capability, Customer Agility, and Organization Performance: A Dynamic Capability Perspective.

[14] Jeble, S., Dubey, R., Childe, S. J., Papadopoulos, T., Roubaud, D., \& Prakash, A. (2018). Impact of big data and predictive analytics capability on supply chain sustainability. The International Journal of Logistics Management, 29(2), 513-538.

[15] Jeble, S., Kumari, S., \& Patil, Y. (2016). Role of big data and predictive analytics. International Journal of Automation and Logistics, 2(4), 307-331.

[16] Lamba, H. S., \& Dubey, S. K. (2015). Analysis of requirements for big data adoption to maximize IT business value. Paper presented at the 2015 4th International Conference on Reliability, Infocom Technologies and Optimization (ICRITO)(Trends and Future Directions).

[17] Lazăr, S. (2016). Determinants of firm performance: evidence from Romanian listed companies. Review of Economic and Business Studies, 9(1), 53-69.

[18] Lee, H., Kweon, E., Kim, M., \& Chai, S. (2017). Does Implementation of Big Data Analytics Improve Firms' Market Value? Investors' Reaction in Stock Market. Sustainability, 9(6), 978.

[19] Mikalef, P., Pappas, I. O., Krogstie, J., \& Giannakos, M. (2017). Big data analytics capabilities: a systematic literature review and research agenda. Information Systems and e-Business Management, 1-32.

[20] Opresnik, D., \& Taisch, M. (2015). The value of big data in servitization. International Journal of Production Economics, 165, 174-184.

[21] Popovič, A., Hackney, R., Tassabehji, R., \& Castelli, M. (2018). The impact of big data analytics on firms' high value business performance. Information Systems Frontiers, 20(2), 209-222.

[22] Raguseo, E., \& Vitari, C. (2018). Investments in big data analytics and firm performance: an empirical investigation of direct and mediating effects. International Journal of Production Research, 1-16. 
[23] Selvam, M., Gayathri, J., Vasanth, V., Lingaraja, K., \& Marxiaoli, S. (2016). Determinants of firm performance: A Subjective Model. Int'l J. Soc. Sci. Stud., 4, 90.

[24] Srinivasan, U., \& Arunasalam, B. (2013). Leveraging big data analytics to reduce healthcare costs. IT professional, 15(6), 21-28.

[25] Wamba, S. F., Akter, S., Edwards, A., Chopin, G., \& Gnanzou, D. (2015). How 'big data'can make big impact: Findings from a systematic review and a longitudinal case study. International Journal of Production Economics, 165, 234-246.

[26] Wamba, S. F., Gunasekaran, A., Akter, S., Ren, S. J.-f., Dubey, R., \& Childe, S. J. (2017). Big data analytics and firm performance: Effects of dynamic capabilities. Journal of Business Research, 70, 356-365.

[27] Yu, W., Chavez, R., Jacobs, M. A., \& Feng, M. (2018). Data-driven supply chain capabilities and performance: A resource-based view. Transportation Research Part E: Logistics and Transportation Review, 114, 371-385. 